\title{
Editorial
}

\section{Traducción de los cuestionarios para su uso en investigación multicultural - estamos haciendo lo correcto?}

\author{
Leonardo Oliveira Reis \\ División de Urología de la Facultad de Medicina de la Universidad de Campinas, Unicamp, Sao Paulo. Brasil.
}

\section{Resumen}

La traducción de instrumentos relacionados con la calidad de vida (HRQL) para su uso en otra cultura es aún una ciencia en desarrollo y hay situaciones de más de una versión del mismo cuestionario. Los temas médicos especializados son áreas de contenido difícil y los buenos traductores profesionales son a menudo incapaces de traducir material médico. Las trampas técnicas pueden conducir a conclusiones erróneas que, aunque, se deban a fallos metodológicos, son indetectables como tales, y se consideran de naturaleza sustantiva, e incluso aquellos que son conscientes del problema encuentran su solución desalentadora. Aunque las normas para la traducción de los cuestionarios no existen, ¿estamos haciendo lo correcto?

Palabras clave: Investigación multicultural. Cuestionario. Traducción.

\section{Translating questionnaires for use in crosscultural research - are we doing the right thing? Abstract}

The translation of health related GoL (HRQL) instruments for use in another culture is still a science under development and there are situations of more than one version of the same questionnaire. Specialized medical subjects are difficult content area and good professional translators are often incapable of translating medical material. Technical traps may lead to erroneous research conclusions that, although due to methodological flaws, are undetectable as such and considered to be substantive in nature and even those who are aware of the problem find its solution daunting. Although standards for translation of questionnaires do not exist, are we doing the right thing?

Keywords: Crosscultural research. Questionnaire. Translating.

$\mathrm{C}$ uando las compañías farmacéuticas evalúan nuevos medicamentos en ensayos clínicos en diferentes países, como es el caso de los organismos reguladores, necesitan garantías de que la auto-evaluación de los pacientes de su condición y los efectos del tratamiento son válidos en los diferentes países, con independencia de sus diferencias lingüísticas y culturales $^{1}$. La Sociedad Internacional de Continencia recomendó la inclusión de las normas de calidad de vida (QoL) en todos los estudios de incontinencia urinaria, y se han desarrollados herramientas de calidad de vida enfermedad-específica ${ }^{2}$.

Debido a la excepcional complejidad, aplicar la metodología estadística adecuada y obtener unos buenos resultados están lejos de ser el principal objetivo en la investigación transcultural de la cali- dad de vida. El objetivo principal es especificar los aspectos culturales y multiculturales como factores distintivos que enriquecerían el modelo y la descripción de las diferencias culturales ${ }^{3}$.

El cuestionario debe ser el mismo en todas las culturas e implica una necesidad de establecer si el concepto existe y se interpreta de manera similar en las dos culturas y, en caso afirmativo, el grado en que se produzca $^{4}$. La duplicación exacta con un lenguaje idéntico y fiel al documento original podría no resultar ser el instrumento apropiado culturalmente. La equivalencia conceptual se entiende por la misma relación con el concepto subyacente en ambas culturas en cuanto a los dominios incluidos y al énfasis dado. Se requiere investigación para determinar cómo la salud y la QoL se conceptualizan en otras culturas. 
El objetivo es alcanzar cuatro tipos de equivalencia: semántico ( $p$ ej, la equivalencia en el significado de las palabras), idiomático (es decir, se han de encontrar expresiones equivalentes o sustituir algunos temas), empírico (p. ej., la situación evocada o representada en la versión original debería encajar en el contexto cultural destino) y conceptual (p. ej., ¿es el concepto explorado válido en la cultura destino?) ${ }^{1}$.

La traducción literal no ha logrado capturar el espíritu de la comunicación o ha sido demasiado libre, sin dedicar suficiente atención a los matices culturales, frases coloquiales, argot y jerga, expresiones idiomáticas, y términos emocionalmente evocadores. Por lo tanto, examinar la equivalencia conceptual no es posible simplemente a través de la traducción y el análisis post hoc de los resultados de cuestionario $^{5}$.

Guyatt GH (1993) proponer un enfoque de "motivos de" en los que los investigadores construyan o revisen un instrumento basado en los hallazgos centrados en grupos analizados de la cultura de interés o, al menos, una estrategia intermedia, utilizando métodos tales como grupos de análisis para investigar tanto la estructura y el contenido del cuestionario propuesto ${ }^{6}$.

Aunque un enfoque conservador en el que los investigadores reproducen (traducen) el cuestionario original en idioma inglés, es una estrategia de ahorro de recursos, y es el método más común de preparación de instrumentos para la investigación multicultural, la opinión del investigador es un elemento importante para tratar de minimizar esos errores. Hay trampas que amenazan la efectividad y algunos de estos problemas son difíciles de detectar y puede tener efectos perjudiciales en los resultados del estudio. Por otro lado, la utilización del mismo instrumento puede unificar la conceptualización del fenómeno estudiado a través de diferentes estudios, y por tanto, los resultados pueden ser comparados ${ }^{1}$.

La traducción de instrumentos relacionados con la salud (QoL) (HRQL) los instrumentos para su uso en otra cultura es aún una ciencia en desarrollo y hay situaciones de más de una versión del mismo cuestionario. ¿Estamos haciendo lo correcto?

\section{Trampas técnicas en la traducción de cuestionarios}

Aunque no existen normas para la traducción de los cuestionarios, hay algunos errores importantes que podrían obstaculizar la adecuada convalidación en la investigación multicultural, la mayoría de ellos en relación con el proceso de traducción.

La metodología de la retraducción podría ser mejor que la simple traducción, pero no garantiza la equivalencia cultural de lo que se traduce. Puede ocurrir porque los retraductores pueden, efectivamente, y de forma intuitiva dar sentido a un lenguaje pobre, corrigiéndolo. También pueden mantener la forma gramatical de la lengua original en la traducción, realizando retraducción gramaticalmente fácil, pero, al mismo tiempo, enmascarando las diferencias importantes entre las dos versiones. Algunos aspectos culturales de la QoL debe ser considerados, incluso si estos aspectos no existen en las actuales versiones en idioma inglés de los instrumentos. El objetivo debe ser identificar lo que es esencial incluir en un instrumento de la $\mathrm{QoL}$ en una población no-inglés parlante para evaluar los temas de particular interés en una determinada cultura. Puede haber diferencias entre las diferentes subculturas que podrían ser abordadas en el cuestionario ${ }^{2}$.

El uso de cualquier instrumento más allá de las muestras con las que se probó inicialmente, sobre todo si las nuevas muestras son multiculturales, presenta retos considerables. Los pacientes bilingües no son plenamente representativos de los monolingües y los cuestionarios traducidos están, con frecuencia, destinados a ser utilizados por los pacientes monolingües. Los ajustes culturales con mecanismo de compensación para los problemas de comprensión entre las culturas y la adaptación de conceptos podrían producirse en su opinión. Las personas bilingües suelen adoptar algunos de los conceptos, valores, actitudes, expectativas y el papel de la cultura de la segunda lengua que ellos han llegado a dominar. Así pues, los bilingües representan una población cuyas respuestas no pueden ser automáticamente generalizadas con las de la población monolingüe ${ }^{2}$. Un sesgo de memoria puede justificar el acuerdo entre 2 versiones. Existe una tendencia a mantener la misma respuesta ya que la misma pregunta se puede identificar por algunas palabras iniciales teniendo en cuenta que los pacientes son bilingües. Estos sesgos contribuyen a un falso nivel más alto de concordancia entre las dos versiones, a pesar de la aleatorización para completar primero las diferentes versiones.

La divulgación de información relacionada con la salud puede ser más aceptable en algunas culturas, 
que, abiertamente abordan las cuestiones mientras que los participantes en otras culturas pueden vacilar ante la revelación a causa de los tabúes. En algunas culturas las diferencias entre excelente, bueno, normal y malos en la evaluación de salud no fueron equivalentes a las diferencias en los Estados Unidos. El formato de puntuación también puede ser inadecuado cuando se encuentran muchas puntuaciones en los extremos de la escala, demostrando que estas opciones tienen un formato inadecuado en estas culturas ${ }^{3}$.

A menos que estos posibles problemas se aborden, los resultados de su investigación pueden ser sospechosos. Hay ejemplos en la literatura del proceso de convalidación que muestran una traducción bien hecha, y, sin embargo, cuando el cuestionario se utilizó en un estudio piloto, los pacientes no entendieron la pregunta. La traducción era buena, pero el tema era culturalmente irrelevante y se tuvo que suprimir o reformular para reflejar la realidad cultural o era, literalmente, bueno, pero confuso cuando se aplicó a un grupo culturalmente distinto ${ }^{4}$. Los temas médicos especializados son áreas de difícil contenido y los buenos traductores profesionales son a menudo incapaces de traducir material médico. Estas trampas técnicas pueden conducir a conclusiones erróneas que, aunque, se deban a fallos metodológicos, son indetectables como tales, y se consideran de naturaleza sustantiva, e incluso aquellos que son conscientes del problema encuentran su solución desalentadora.

\section{REFERENCIAS}

1. Acquadro C, Conway K, Hareendran A, Aaronson $\mathrm{N}$ for the European Regulatory Issues and Quality of Life Assessment (ERIQA) Group. Literature Review of Methods to Translate Health-Related Quality of Life Questionnaires for Use in Multinational Clinical Trials Value in Health. 2008;11(3):509521.

2. Barber MD, Kuchibhatla MN, Pieper CF, Bump RC. Psychometric evaluation of comprehensive condition-specific quality of life instruments for women with pelvic floor disorders. Am J Obstet Gyencol. 2001;185(6):1388-1395.

3. Sperber AD. Translation and Validation of Study Instruments for Cross-Cultural Research, Gastroenterology 2004;126(1 Suppl 1):S124-128.

4. Herdman M, Fox-Rushby J, Badia X. A model of equivalence in the cultural adaptation of HRQoL instruments: The universalist approach. Quality of Life Research. 1998;7(4):323-325.

5. Sperber AD, DeVellis RF, Boehlecke B. Cross-cultural translation: Methodology and validation. J Cross-Cult Psicol. 1994;25: 501-524.

6. Guyatt GH. The philosophy of health-related quality-of-life translation. Quality of Life Research. 1993;2(6):461-465.

Correspondencia autor: Dr. Leonardo Oliveira Reis División de Urología. Universidad Estatal de Campinas Unicamp, Sao Paulo. Brasil

R. Votorantim, 51, ap. 43, Campinas-SP, Brasil - 13073-090

Tel./Fax: + 5519 3284-4420

E-mail autor: reisleo@unicamp.br

Información autor: Editorial

Trabajo recibido: octubre 2008 